Am. J. Trop. Med. Hyg., 71(Suppl 2), 2004, pp. 156-161

Copyright (C) 2004 by The American Society of Tropical Medicine and Hygiene

\title{
THE SOCIAL BURDEN OF MALARIA: WHAT ARE WE MEASURING?
}

\author{
CAROLINE O. H. JONES AND HOLLY A. WILLIAMS \\ Department of Infectious and Tropical Diseases/Disease Control and Vector Biology Unit, London School of Hygiene and Tropical \\ Medicine, London, United Kingdom; Malaria Epidemiology Branch, Centers for Disease Control and Prevention, Atlanta, Georgia
}

\begin{abstract}
Definitions of the burden of malaria vary by public health discipline. Epidemiologists and economists commonly use a quantitative approach to measure risk factors and associate them with disease outcomes. In contrast, since burden is itself a cultural construct, an anthropologic perspective of the burden of disease considers the sociocultural context in which these risk factors exist. This broader concept of burden is rarely tackled, most likely stemming from a lack of understanding of what is meant by the term social burden. This report describes the concept from an anthropologic perspective. The aim is to provide a better understanding of the process through which social and cultural factors affect the biomedical burden of malaria. The consequences of adopting this perspective for public health in general and malaria interventions in particular are discussed.
\end{abstract}

\section{INTRODUCTION}

The global impact of malaria is staggering, regardless of how it is measured. Biomedically, the burden of disease is a function of many things and various public health disciplines define it differently. Epidemiologists refer to burden in terms of morbidity and mortality, while economists refer to a quantification of the costs (direct and indirect), and effects on levels of productivity, national growth, and development. Common to both of these approaches is a focus on disease and risk factors, with the aim of establishing causative linkages with broad quantitative outcomes. However, burden of disease is also a function of factors in the social realm, an area that, while alluded to frequently as a concept, is, in practice, commonly ignored.

Perhaps one of the reasons that in practice the social factors are often ignored is that it is unclear what is meant by the social burden of malaria. Our aim in this paper is to try to understand what is meant by the term. Can it be considered as a universal quantifiable entity unto itself, or on the other hand, is it more of a process that links to other outcomes, such as the biomedical burden of the disease?

\section{BACKGROUND}

In reviewing the literature for this paper, we found that although there is debate about the most appropriate methods for quantification, the economic burden of malaria has been frequently studied and is generally is well described. ${ }^{1-3}$ However, the term social burden of malaria is rarely mentioned in the literature, much less described or analyzed. In the one paper that discusses both the economic and social burden, ${ }^{1}$ it is suggested that the social burden of malaria is the cost to society imposed by the existence of the disease. The authors of this paper focus on identifying those economic costs, which are frequently ignored by traditional economic burden studies with the aim of quantifying their impact on economic growth and productivity. They suggest that the disease causes changes in household behaviors that result in broad social costs, such as decisions related to reproduction, education, and economic matters, which, in turn, have a long-term effect on economic growth and development. This approach to social burden is, therefore, fundamentally an economic approach, using the term social as a subset of economic.

A major concern with adopting this narrow approach to the concept of social burden is that other factors that contribute to the social burden of the disease will be ignored. Such factors include the influences of culture, beliefs, and political context that are known to affect perceptions, individual behavior, social structure, and social action. In recent years, there has been growing recognition among public health practitioners, and others involved in disease control programs, that such sociocultural factors significantly influence the observed distribution of health and illness, and that issues of power relationships, inequity, marginalization, and racism affect how diseases are created, distributed, and treated. ${ }^{4}$ These factors will all have a significant effect on the nature, perceived size, and distribution of the burden of disease.

However, in the context of malaria control, the term social burden remains nebulous. Few people appear to have a clear definition of what is meant by the term. In a straw poll of public health colleagues, when asked "what is the social burden of malaria," the most common response was "what do you mean?" Social burden is more than social cost but we have no clear consensus on its definition.

\section{THE CONCEPT OF SOCIAL BURDEN}

Biomedicine is based on positivist assumptions, that is, the premise of universal scientific truths that can be observed, measured, and quantified. From a sociocultural perspective, these assumptions are inappropriate and hinder attempts to interpret and understand social and cultural phenomena. This does not mean that we are not interested in universals, but they may be different from those that are assumed from a positivist perspective. Using a sociocultural perspective, there are no universal truths since the origin and significance assigned to any event is modified by the social context in which it occurs. As a consequence, unlike the concept of biomedical burden, perceptions of social burden vary among societies, cultures, individuals, and even within an individual over time. The concept is individually defined and modified by social, cultural, and political influences.

While the concept of social burden is, therefore, nonquantifiable, it is essential to furthering our understanding of locally appropriate and acceptable strategies to reduce the biomedical burden of malaria. Understanding perceptions of the burden of malaria (its social components and consequence) is as important as understanding and quantifying the biomedical burden, since perceptions form the basis for ac- 
tion. We cannot quantify social burden, but we can seek to understand it.

To address the issue of the social burden of malaria we have therefore adopted an anthropologic approach whereby the aim is to better understand the process through which social and cultural factors affect the biomedical burden of malaria. Anthropologists are generally "concerned with meanings rather than measurements" 5 and so we seek to describe and understand what malaria means: how local perceptions of the disease, cultural norms, and beliefs, as well as the social, economic, and political context, influence behaviors, and how these link to the quantifiable biomedical outcomes. We are interested in investigating the effect of social organization and cultural norms and beliefs on the development of disease and the observed distribution of the malaria burden (e.g., burden implying all components, such as mortality, morbidity, economic costs, etc.).

In other words, while biomedical factors, such as endemicity, underlying health status, and immunologic status, all contribute to the burden of malaria, these factors alone cannot account for the observed biomedical disease burden. Focusing on the quantification of the burden puts disease at the center of enquiry, ignores ordinary life, and tends to overlook the complex interrelations that shape the health of populations living in malaria-endemic regions.

\section{ANTHROPOLOGIC PERSPECTIVE}

It has long been recognized that non-biomedical factors, such as socioeconomic status, play a significant role in the distribution of the burden of disease. ${ }^{6-8}$ During the 1970 s, the acceptance of the use of social science in public health increased. $^{9}$

Since the 1980s, anthropologists have been used in public health research and implementation programs primarily to assist in designing more culturally appropriate messages to promote individual and community level acceptance of interventions. Attention has focused on the investigation of traditional folk beliefs and local perceptions about disease causation and treatment. ${ }^{10-12}$ However, the rationale underlying the employment of social scientists in public health has frequently been based on the assumption that knowledge of etiology is the fundamental factor in behavior change. Local etiology is important and has been shown, in some cases, to influence treatment seeking behavior, but it is by no means the only influence, and folk diseases in which etiologic concepts are the main trigger for choice of care are rare. ${ }^{13}$ There is also a large body of data suggesting that simply giving people scientifically correct information with no additional inputs (even in a locally appropriate manner) is unlikely to influence their actual behavior. ${ }^{14}$ While correct biomedical knowledge may be accepted and believed, it may not completely replace traditional knowledge. Rather, the biomedical knowledge may co-exist with traditional concepts or be incorporated into a local model that best fits with local experience. ${ }^{15}$ As a consequence, provision of knowledge may not result in the expected behavior change.

Much recent research has shown that health behaviors are not simply a function of knowledge or beliefs but are also modified and constrained by the social, cultural, economic, and political context in which they occur. ${ }^{16-18}$ While society's perceptions of disease, malaria, and perceived risk influence people's willingness to change their health behaviors, the political and economic context, social organization, and cultural rules regarding roles and expected behaviors affect their ability to change these behaviors. As such, sociocultural factors, such as social organization, culturally defined social roles, and cultural beliefs about disease, all play an important role in the observed variability in the disease burden. It is only when cultural perceptions of illness are considered in conjunction with the social context in which they occur that we can start to understand human illness behaviors and their consequences for observed patterns of disease.

Influence of social roles and cultural expectations. So what do we mean by cultural rules regarding roles and behaviors? Among anthropologists, the term culture generally refers to a shared conceptual code, a code of shared rules, and common meaning. A society is a population that shares a common culture and who organize themselves into various groups, hierarchies, and roles based on this code. ${ }^{19}$ The members of these groups share a social identity and, in any given society, the behavior of an individual is mediated by their social identity. That is, social identities are associated with particular roles within the society and these roles are associated with a range of expected norms of behavior. ${ }^{20}$ Individuals move among roles throughout their lives and, within a society, the behaviors associated with particular roles also change over time (that is, neither culture nor social organization are fixed elements. Rather, they are both dynamic systems affected by intrinsic and extrinsic pressures).

Much of our behavior is influenced by our particular social identity and social identity is proscribed by many factors such as sex, age, socioeconomic status, class, ethnicity, and even political affiliation (Figure 1).

In the following sections, we illustrate how normally expected behaviors interact with perceptions of disease to affect what people do when they become sick.

Perceptions of disease and the accepted sick role. Malaria, as defined biomedically, is a specific disease with a particular etiology and associated signs and symptoms. However, becoming ill with any disease is a subjective process involving the interpretation of the origin and the significance of the symptoms. These perceptions affect behavior relating to the illness and contribute to shaping the meaning individuals give to the experience. ${ }^{21}$ In every society, there are socially acceptable behaviors associated with being sick, and specific illnesses are often related to particular culturally acceptable behaviors or sick roles for that illness. The sick role is a social process that is governed by the cultural norms of each society. ${ }^{22}$ In other words, in every society there are social conventions surrounding how people should behave when they become ill. Social and cultural factors determine which and when symptoms are abnormal or serious enough to frame a response. The acceptable sick role for a particular disease depends partly on the perceived relative risk of the disease, both to the individual and to the health of the community.

The sick role and uncomplicated malaria. In most endemic areas in sub-Saharan Africa, the term malaria is often used either alone or in conjunction with a local term for fever (e.g., homa in KiSwahili) to refer to a constellation of symptoms that frequently correspond closely with clinically defined mild or uncomplicated malaria. ${ }^{23-26}$ The illness malaria is perceived by most adults as a common illness with a range of signs and symptoms that are seen as within tolerable limits, 

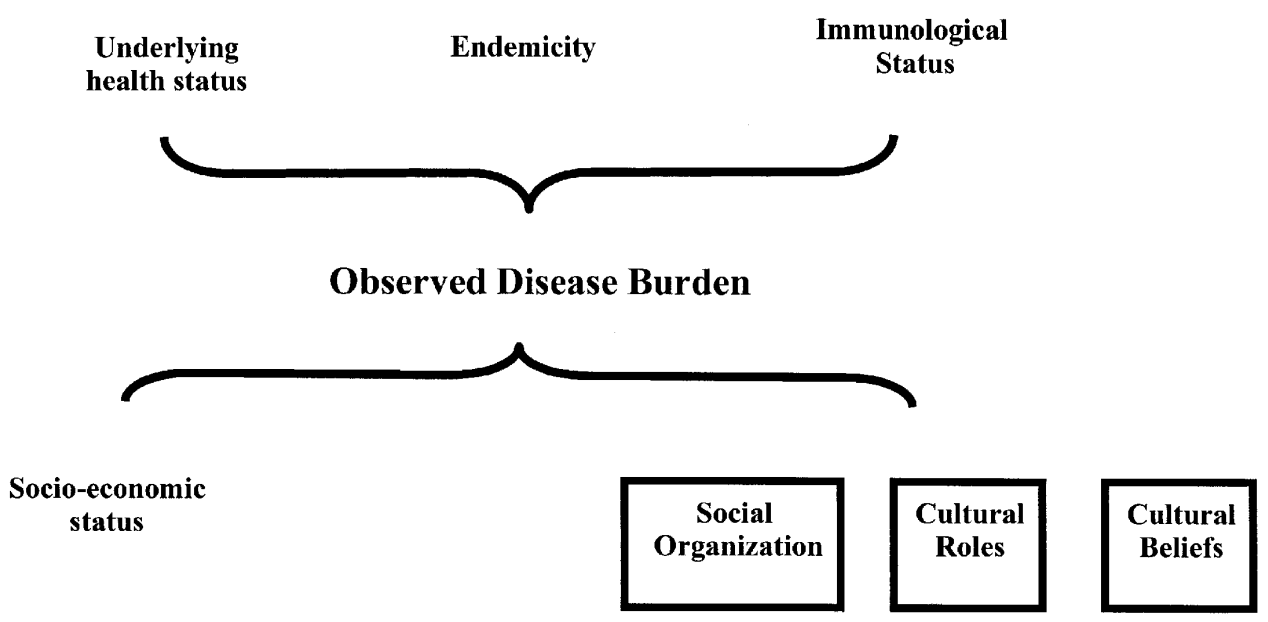

FIGURE 1. Factors influencing the burden of malaria.

which cause minimal disruption to their lives. Even among children, uncomplicated malaria is frequently seen as a mild everyday illness that is not preventable but treatable. ${ }^{27}$ In this context, uncomplicated malaria is therefore socially defined as a normalized illness, with treatment being the responsibility of the individual or close family. ${ }^{28}$ In addition, unlike diseases such as human immunodeficiency virus/acquired immunodeficiency syndrome (HIV/AIDS) or tuberculosis, there is no stigma attached to uncomplicated malaria. It is a wellknown and socially acceptable disease, with no social sanctions or associated emotional trauma from stigmatization linked to its presence. As a result, there is low social pressure to seek treatment, provide money for treatment of close relatives, or even to comply with completing malaria treatment.

As previously mentioned, the way in which an illness is perceived has consequences for the sick role that the ill person is expected to adopt. One of the consequences of viewing uncomplicated malaria as a normalized illness is that people with the illness are not expected to create a huge fuss or deviate significantly from everyday behavior. However, the accepted sick role for the illness is also affected by the social position and expected social role of the person with the illness. For example, what is acceptable behavior for men may not be acceptable for women. In many countries, men may be expected (and have the capacity) to seek treatment quickly so that they can maintain their roles as breadwinners for the family. On the other hand, women are frequently expected to continue in their primary roles as caregivers and they often do not have the resources to make unilateral treatment seeking decisions. They commonly have to consult others in the household about their treatment-seeking decisions and if they admit illness and seek treatment of a common illness such as malaria, this may be considered a sign of weakness. The social standing for such women and their children may be decreased with possible subsequent impact on their long-term welfare. ${ }^{29}$

The sick role and severe or complicated malaria. In contrast to uncomplicated malaria, the disease that is biomedically defined as severe or complicated malaria is often perceived by communities in sub-Saharan Africa to be unrelated to the mild disease.

Symptoms such as splenomegaly, anemia, and, in particular, convulsions are rarely associated with the disease malaria and are frequently thought to be of supernatural or spiritual origin. $^{24,30,31}$ Illnesses of this supernatural (rather than normalized) type are perceived as social in nature and a threat to the larger community. That is, these symptom constellations are a sign that something is wrong in the community and, unless dealt with, others in the community may be at risk. ${ }^{28}$ Given the social interpretation of these symptoms, there is considerable social pressure to include a wider group (such as mothers-in-law or elders) in treatment decisions. While on the surface it may appear that having a supernatural illness, such as severe malaria, means that greater resources can be mobilized, it also means that the sick individual (or their caregiver) loses control over the decision-making process.

For example, a family has a child with a high fever that is starting to have a seizure. The norm for the village in which they live is to use the services of a traditional healer before visiting the hospital. However, they choose to take action that runs counter to expected behaviors, and they take the child to the hospital. If the child dies, they may be socially isolated due to having ignored the social rules governing behavioral expectations in this specific situation. The outcomes that they face may cause the family additional financial obligations because they are unlikely to receive the community support that generally occurs at the time of a death. This may also exaggerate the grieving process because they are mourning the loss of the child, as well as the loss of accustomed and expected social support from their community.

These examples demonstrate that perceptions of disease interact with a person's sociocultural reality (their social role and expected behaviors) to shape both behavior and ability to respond to disease. It is the interaction between the expected behavior and perceptions of disease, as defined individually and by society, that affects both if and how an individual acts to prevent disease, as well as what they do when they become sick (their illness behavior). It is these behaviors that influence the distribution of the biomedical burden of disease or disease outcomes.

Social vulnerability. In any society, there are groups of people who, because of their socially and culturally defined roles, have limited control over their ability to admit to illness, mobilize resources, access services, and make decisions. This lack of power and personal control places them in a position in which they can be considered to be socially vulnerable. The term social vulnerability was first used in the 
early-mid 1990s by social scientists working in the HIV/AIDS arena to highlight the importance of social factors that make some groups or individuals more susceptible to infection, and more limited in their ability to respond to illness than others. $^{32}$ The notion of vulnerability draws attention to the factors that create inequality and allows us to broaden our focus from individual factors creating the burden to a consideration of the wider social, economic, and political factors that shape the distribution of the burden of disease. A diagrammatic representation of the factors affecting social vulnerability and their possible outcomes is shown in Figure 2. Social vulnerability has a significant impact on illness behaviors, as described below.

As previously described, in many societies women may be constrained in their ability to admit to being ill for fear of being thought weak. On the other hand, men may be unable to admit to illness and cannot take time off work for fear of losing their job. The mobilization and partitioning of resources is also frequently influenced by the social position of an individual within a household (or a household within a community). ${ }^{28}$ For example, money for the treatment of uncomplicated malaria may be provided more easily and rapidly for the eldest son in a household than for an orphan being raised in the household of a relation.

The ability to access health care and other services is also frequently influenced by sociocultural considerations. In some societies, women are unable to access health care due to cultural constraints on their movement and interaction with men, ${ }^{33}$ and in others certain ethnic, social, or political groups may be excluded from public health care ${ }^{34}$ or discouraged from accessing other public health goods. ${ }^{35}$

Societal rules that govern behavior directly impact the ability of individuals and groups to make health care decisions. For example, picture a young mother whose husband is away working. Her child becomes sick with a fever and she wants to take the child to a health center. Societal rules dictate that a young woman or mother should seek the advice of more senior women. She seeks their advice, but co-wives or her mother-in-law tell her she must wait for the husband to return to make this decision. At this juncture, she has two choices that she can make in terms of treatment-seeking decisions. The first choice is that she can listen to the advice of the more senior women and wait. With this choice, she is seen as compliant with the expected social norms, but she risks the health of her child. If the child becomes sicker, she may also be judged for not preventing the illness. The second choice is that she decides to ignore their advice and she, independently, makes a decision and takes the child for treatment. Scenario one is that the child recovers. In this situation, the mother may be scolded and the position and welfare of herself and her child (and any other children she may have) in the household may decrease temporarily because she disobeyed the normative rules. In the worse case scenario of the child dying, the mother is likely to be scorned because she not only disobeyed, but the child died. She faces divorce, may be forced to return to her village of birth, and faces shame, increased dependency, and even lower social status for herself and any other children she may have. ${ }^{36}$ Thus, in negotiating action for a specific illness episode, the mother needs also to take into consideration the long-term welfare of herself and any other children she may have.

In the situations described above, the individuals have limited power or influence over decisions that affect their capacity to respond to illness. That is, they can be considered the socially vulnerable.

Socially vulnerable will vary from society to society and, most often, represent the truly marginalized sectors of any population: marginalized economically, socially, and/or politically. From an intervention perspective, the results of such vulnerability is that some groups in the population, due to their social position, may have the willingness to change but not the ability to respond. Behaviorally, this translates into outcomes such as having limited knowledge about appropriate treatment, needing to delay seeking treatment when illness is recognized (limited options available to them), or having little or no access to preventive measures.

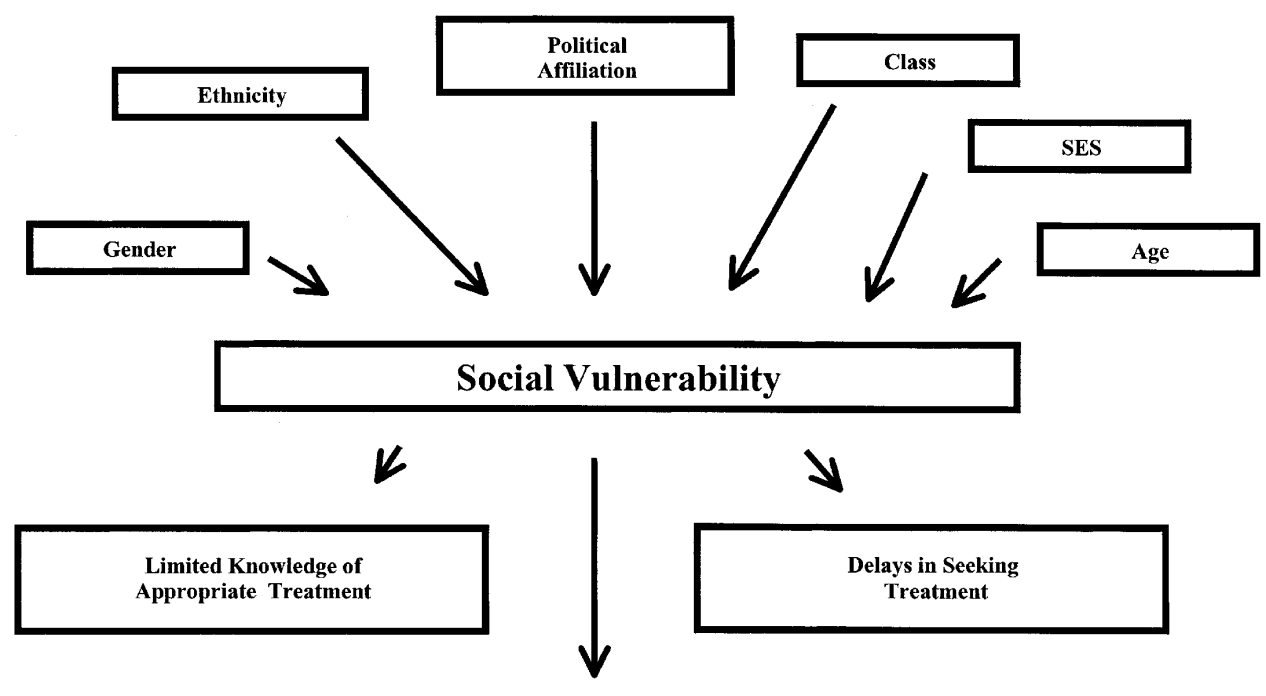

Inability to Access Preventive Measures Due to Costs or Acceptability

FIGURE 2. Social vulnerability. SES = socioeconomic status. 
Social burden: intervention and control. From a practical point of view, if we adopt this broader sociocultural perspective to the burden of malaria, we can start to identify those groups in any society or community that are the most socially vulnerable to the burden of malaria, and understand how the biomedical burden of malaria relates to other burdens (social, political, and economic) that people suffer in their day-to-day lives that affect their prevention and treatment-seeking activities. Adopting this perspective then necessitates changing our approach to intervention development.

With existing interventions, we must reconsider whom we are trying to target and who has the ability or social power to act or affect change. For example, in terms of selling insecticide-treated mosquito nets, campaigns need to target those individuals who control the finances and make the financial decisions, rather than simply targeting women, because many women are not in a position to make the decision to buy them. Also related to expenditure, in an atmosphere of cost recovery and user fees, we need to understand from communities and households how decisions are made regarding health expenditures, both to identify the decision makers as targets for information, and to identify those who may be most disadvantaged by this approach to intervention delivery.

In relation to this, we also need to consider how easy or difficult it is for interventions to be incorporated within the existing social structure and cultural norms. Is it likely that the invention will require the adoption of behaviors that run counter to current sociocultural norms? Or can we adapt interventions that acknowledge parallel belief systems about disease? Taking this approach, rather than offering interventions that compete with local knowledge and societal norms, it then becomes important to intervene in ways that enhance the participation of those most affected. To be successful and sustainable with our interventions, we should broaden our thinking to incorporate community priorities in developing interventions that address the concerns both of the communities, as well as those of public health practitioners. This means that community and individual perceptions about the burden of malaria must be understood.

Approaches to tackling the burden of malaria have changed. In 1978, the Alma-Ata Declaration advocated the adoption of community-based health care. The concern was for universal coverage and provision of basic services through a multi-sectoral approach. ${ }^{37}$ Today, in contrast, the Global Fund to fight AIDS, Tuberculosis and Malaria (GFATM) concentrates on reducing the burden of disease by funding disease specific interventions with the expectation that this approach will contribute to strengthening the health care system. However, as several investigators ${ }^{38-40}$ have noted, in view of the status of the health infrastructure and economic situation of most GFATM target countries, this approach is likely to be ineffective in producing sustainable reductions in the disease burden without specific support to infrastructure and capacity development. In addition to these concerns, if efforts to reduce disease burden are concentrated solely in the health care sector, this fails to address the fact that the health of an individual and/or community is shaped by other influencing factors or burdens (e.g., sociocultural norms, housing, food security, employment, land tenure). ${ }^{41}$

Malaria does not stand alone as an isolated issue in peoples' lives. In fact, as public health practitioners, we have limited understanding of the weight that malaria holds in relation to other pressing concerns at any given time. Recognizing this, we need to shift from solitary to integrated designs of interventions that are placed within the broader social, cultural, political, and economic context. Malaria control interventions should reflect a wider development perspective. We need to return to the historically broad concept of public health, whereby the underlying determinates of health and disease are cornerstones of the public health discourse. Instead of increasing competition for funding among the three GFATM diseases, ${ }^{42}$ the Board of the GFTAM should be specifically supporting the development of health sector capacity and advocating for approaches to public health that recognize the influence of other sectors. By enhancing the overall ability of the health care sector to provide adequate preventive and curative measures and by improving individuals' abilities to access such care, the burden of malaria in its widest sense should be lessened. Perhaps it is time to move away from the concept of "how can we get them to..." and start thinking about "how can we help people increase their capacity to...?"

\section{CONCLUSIONS}

In conclusion, the social burden of malaria remains an elusive concept. It is not a universal entity that can be measured and quantified. It is, however, a process that can be understood and linked to outcomes such as morbidity and mortality. Those who have the least amount of social influence and power, the socially vulnerable within a society, share a larger burden of disease, no matter how disease burden is measured.

If interventions are conceived and developed from purely a technical perspective, can we realistically expect to be successful in reducing the burden of malaria? With continued commitment from those working directly in malaria control initiatives, such as Roll Back Malaria and improved resources, we can move toward success. Our movements will be strengthened if we include in our approach a focus on the broader social, political, cultural, and economic context in which malaria occurs.

Received August 21, 2003. Accepted for publication January 8, 2004.

Acknowledgments: We acknowledge the support of the Department for International Development Malaria Knowledge Program at the London School of Hygiene and Tropical Medicine, the Malaria Branch of the Centers for Disease Control and Prevention (in particular Dr. Peter B. Bloland for his helpful comments), and the Partnership for Social Sciences in Malaria Control.

Authors' addresses: Caroline O. H. Jones, Department of Infectious and Tropical Diseases/Disease Control and Vector Biology Unit, London School of Hygiene and Tropical Medicine, Keppel Street, London WC1E 7HT, United Kingdom, Telephone: 44-20-7927-2649, Fax: 44-20-7580-9075, E-mail: caroline.jones@1shtm.ac.uk. Holly A. Williams, Malaria Epidemiology Branch, Centers for Disease Control and Prevention, Mailstop F-22, 4770 Buford Highway NE, Atlanta, GA 30341, Telephone: 770-488-7764, Fax: 770-488-7794, E-mail: hbw2@cdc.gov.

\section{REFERENCES}

1. Sachs J, Malaney P, 2002. The economic and social burden of malaria. Nature 415: 680-685.

2. Gallup J, Sachs J, 2001. The economic burden of malaria. Am J Trop Med Hyg 64 (Suppl): 85-96

3. Chima RI, Goodman C, Mills A, 2002. The economic impact of malaria in Africa: a critical review of the evidence. Health Policy 63: 17-36. 
4. Heggenhougen K, 2000. More than just "interesting!" Anthropology, health and human rights. Soc Sci Med 50: 1171-1175.

5. Keesing RM, 1981. Cultural Anthropology: A Contemporary Perspective. New York: Holt, Reinhart \& Winston.

6. Walley J, Wright J, Hubley J, 2001. Public Health: An Action Guide to Improving Health in Developing Countries. Oxford, United Kingdom: Oxford University Press

7. Baum F, 1999. The New Public Health: An Australian Perspective. Melbourne: Oxford University Press

8. Garrett L, 1994. Thirdworldization: the interaction of poverty, poor housing and social despair with disease. Garrett L, ed. The Coming Plague: Newly Emerging Diseases in a World out of Balance. London: Penguin Books, 457-527.

9. Inhorn MC, Brown PJ, 1997. Introduction. Inhorn MC, Brown PJ, eds. The Anthropology of Infectious Disease: International Health Perspectives. New York: Gordon and Breach, 3-29.

10. Kendall C, Foote D, Martorell R, 1984. Ethnomedicine and oral rehydration therapy: a case study of ethnomedical investigation and program planning. Soc Sci Med 19: 253-260.

11. Mull JD, Mull DS, 1988. Mothers' concepts of childhood diarrhoea in rural Pakistan: what ORS program planners should know. Soc Sci Med 27: 53-67.

12. Gove S, Pelto GH, 1994. Focused ethnographic studies in the WHO program for the control of acute respiratory infections. Med Anthropol 15: 409-424.

13. Kroeger A, 1983. Anthropological and socio-medical health care research in developing countries. Soc Sci Med 17: 147-161.

14. Griffiths M, 1990. Using anthropological techniques in program design: successful nutrition education in Indonesia. Coreil $\mathrm{J}$, Mull JD, eds. Anthropology and Primary Health Care. Boulder, CO: Westview Press, 154-169.

15. Hausmann Muela S, Muela Ribera J, Tanner M, 1998. Fake malaria and hidden parasites: the ambiguity of malaria. Anthropol Med 5: 43-61.

16. Farmer P, 1997. Social science and the new tuberculosis. Soc Sci Med 44: 347-358.

17. Yoder PS, 1997. Negotiating relevance: belief, knowledge and practice in international health projects. Med Anthropol $Q 11$ : 131-146.

18. Wallman S, 1998. Ordinary women and shapes of knowledge: perspectives on the context of STD and AIDS. Public Understanding Sci 7: 169-185.

19. Keesing RM, Strathern AJ, 1998. Cultural Anthropology: A Contemporary Perspective. Fort Worth, TX: Harcourt Brace College Publishers

20. Biddle BJ, 1979. Role Theory: Expectations, Identities and Behaviors. New York: Academic Press, Inc.

21. Helman CG, 1994. Doctor-patient interactions. Helman CG, ed. Culture Health and Illness. Oxford, United Kingdom: Butterworth Heinmann, 101-145.

22. Parsons T, 1958. Definitions of health and illness in the light of American values and social structure. Jaco EG, ed. Patients, Physicians and Illness. Glencoe, IL: Free Press.

23. Agyepong I, Manderson L, 1994. The diagnosis and management of fever at household level in the greater Accra region, Ghana. Acta Trop 58: 317-330.

24. Kengeya-Kayondo J, Seeley J, Kajura-Banjeja E, Kabunga E, Mubiru E, Sembajja F, Mulder D, 1994. Recognition, treat- ment seeking behaviour and perception of cause of malaria among rural women in Uganda. Acta Trop 58: 267-273.

25. Ruebush T, Kern M, Campbell C, Oloo A, 1995. Self treatment of malaria in a rural area of western Kenya. Bull World Health Organ 73: 229-236.

26. Munguti K, 1998. Community perceptions and treatment seeking for malaria in Baringo District, Kenya: Implications for disease control. East Afr Med J 75: 687-691.

27. Mwenesi H, Harpham T, Snow R, 1995. Child malaria treatment practices among mothers in Kenya. Soc Sci Med 40: 1271-1277.

28. Muela SH, Mushi AK, Muela JR, 2000. The paradox of coats and affordability of traditional and government health services in Tanzania. Health Policy Plann 15: 296-302.

29. Munguti K, 1998. Social conditions and cultural beliefs on malaria. Afr J Med Pract 5: 286-288.

30. Ahorlu C, Dunyo S, Afari E, Koram K, Nkrumah F, 1997. Malaria-related beliefs and behaviours in southern Ghana: implications for treatment, prevention and control. Trop Med Int Health 2: 488-499.

31. Makemba AM, Winch PJ, Makame VM, Mehl GL, Premji Z, Minjas JN, Schiff CJ, 1996. Treatment practices for degedege, a locally recognized febrile illness, and implications for strategies to decrease mortality from severe malaria in Bagamoyo District, Tanzania. Trop Med Int Health 1: 305-313.

32. Parker RG, 1996. Empowerment, community mobilization and social change in the face of HIV/AIDS. AIDS 10 (Suppl 3): s27-s31.

33. Tanner M, Vlassoff C, 1998. Treatment-seeking behaviour for malaria: A typology based on endemicity and gender. Soc Sci Med 46: 523-532.

34. Hartigan P, 2001. The importance of gender in defining and improving quality of care: some conceptual issues. Health Policy Plann 16 (Suppl 1): 7-12.

35. Winch PJ, Makemba AM, Makame VR, Mfaume MS, Lynch MC, Premji Z, Minjas JN, Shiff CJ, 1997. Social and cultural factors affecting rates of regular re-treatment of mosquito nets with insecticide in Bagamoyo District, Tanzania. Trop Med Int Health 2: 760-770.

36. Molyneux CS, Murira G, Masha J, Snow RW, 2002. Intrahousehold relations and treatment decision-making for childhood illness: A Kenyan case study. J Biosoc Sci 34: 109-131.

37. Hall JJ, Taylor, 2003. Health for all beyond 2000: The demise of the Alma-Ata Declaration and primary health care in developing countries. Med J Aust 178: 17-20.

38. Brugha R, Starling M, Walt G, 2002. GAVI, the first steps: lessons from the Global Fund. Lancet 359: 435-438.

39. Lambert ML, van der Stuyft P, 2002. Editorial: Global health fund or global fund to fight AIDS, tuberculosis, and malaria? Trop Med Int Health 7: 557-558.

40. Moerman F, Lengeler C, Chimumbwa J, Talisuna A, Erhart A, Coosemans M, D'Alessandro U, 2003. The contribution of health-care service to a sound and sustainable malaria-control policy. Lancet Infect Dis 3: 99-102.

41. Morgan LM, 2001. Community participation in health: perpetual allure, persistent challenge. Health Policy Plann 16: 221230.

42. Teklehaimanot A, Snow RW, 2002. Commentary. Will the Global Fund help roll back malaria in Africa? Lancet 360: 888-889. 